\section{rev Psi}

Revista de Psicología (UNLP)

https://revistas.unlp.edu.ar/revpsi

\title{
Continuidad de los vínculos familiares: impacto de las resoluciones judiciales en violencia familiar
}

\author{
Analía Verónica Losada' \\ Jorge Mario Caruso²
}

\section{Correspondencia}

analia_losada@yahoo.com.ar

Filiaciones institucionales

${ }^{1}$ Universidad de Flores (UFLO, Argentina) $\quad{ }^{2}$ Universidad Austral / Universidad Nacional de (Argentina)

\section{Resumen}

La violencia intrafamiliar es un acontecer psicosocial trascendente para la sociedad en su conjunto. El espacio judicial se constituye como el único recurso, que debería ser el último, de contención y morigeración de los efectos devastadores de la violencia. El objetivo de este trabajo es describir las consecuencias producidas por la intervención judicial en los casos de violencia familiar, sometidos a la consideración de los jueces de familia, sobre la integridad psicosocial del vínculo parental. Se llevó adelante un estudio mixto que incluyó 453 encuestas a universitarios y diez entrevistas en profundidad a jueces. Los resultados muestran que la imposición de la ley es restrictiva respecto de los vínculos familiares y produce efectos negativos en la continuidad de vínculos parentales, siendo los niños los más damnificados y afectados en virtud de su involuntario involucramiento.

\section{Palabras clave}

violencia | familia | vínculos | justicia | parental

Losada, A. V. y Caruso, J. M. (2021).

Continuidad de los vínculos familiares: impacto de las resoluciones judiciales en violencia familiar. Revista de Psicología, 20(2), 55-74. HTTPS://DX.DOI. ORG/10.24215/2422572XE090

\section{Proceso editorial}

Recibido

9 sep. 2020

Aceptado

8 abr. 2021
1 ra decisión

28 mar. 2021

Publicado

9 abr. 2021
ISSN

2422-572X

Licencia

Licencia de Cultura Libre CC-BY 4.0

(Compartir - Adaptar - Atribuir)

Entidad editora

RevPsi es una publicación de la

Facultad de Psicología (Universidad

Nacional de La Plata, Argentina) 


\section{Continuidade dos laços familiares: impacto das decisões judiciais sobre a violência familiar}

\section{Resumo}

A violência intrafamiliar é um grande evento psicossocial para a sociedade em sua totalidade. O espaço judicial é o único meio de conter e mitigar os efeitos devastadores da violência. O objetivo deste trabalho é descrever as conseqüências produzidas pela intervenção judicial em casos de violência familiar, sujeita à consideração dos tribunais de família, sobre a integridade psico-social do vínculo parental. Realizamos um estudo misto entrevistando 453 estudantes universitários, bem como juízes. Os resultados mostram que a imposição da lei é restritiva em relação aos laços familiares e produz efeitos negativos sobre a continuidade dos laços parentais, sendo as crianças as mais afetadas em virtude de seu envolvimento involuntário.

\section{Palavras-chave}

violência | família | vínculos | justiça | parental

\section{Continuity of family ties: impact of court decisions on family violence}

\section{Abstract}

Intrafamily violence is a major psychosocial event for society in its entirety. The judicial space is the only means of containing and mitigating the devastating effects of violence. The purpose of this work is to describe the consequences produced by judicial intervention in cases of family violence, subject to the consideration of the family courts, on the psycho-social integrity of the parental bond. We carried out a mixed study by interviewing 453 university students as well as judges. The results show that the imposition of the law is restrictive with respect to family ties and produces negative effects on the continuity of parental ties, with children being the most affected by virtue of their unwitting involvement.

\section{Keywords}

violence | family | bonds | justice | parental 
Por diversas razones, algunos individuos desarrollan características de descontrol de su agresividad que los lleva a desplegar conductas violentas dentro de su entorno más íntimo, lo que afecta, en diferentes grados, a los integrantes de su núcleo familiar. No quedan espacios exentos ni sitios seguros cuando de violencia se trata, tanto es así que hasta se ha instalado en lo más profundo de las vidas, en los espacios más sensibles para las familias, es decir en los propios hogares. En ese contexto, todos los miembros se interrelacionan con los componentes de las demás células de la comunidad y esto provoca que hechos producidos en la intimidad de los hogares impacten inmediatamente en las esferas sociales.

Decir que la sociedad está enferma de violencia no es novedoso, ya que desde hace mucho tiempo se la padece en casi todos los ámbitos, tanto sea en los estadios de fútbol como en el tránsito, en las manifestaciones públicas, en los establecimientos educativos y hasta en los centros de salud, entre otros (Caruso, 2016).

Bandura y Walters (1987), señalados como los principales defensores de la teoría del aprendizaje social, y Worchel et al. (2002) destacan a los niños como personas que aprenden cómo, cuándo y a quién violentar, que aunque la mayor parte de esas enseñanzas proviene de la expectación de sus padres, también lo hacen de sus compañeros y de las imágenes de violencia emitidas por los medios de comunicación. La agresividad, energía básica para la subsistencia de los seres vivos, debe ser modelada y encauzada por los distintos mecanismos socio familiares y si en determinadas situaciones resulta ilimitada provoca una descarga denominada agresión (Caruso, 2016, 2017a).

En este sentido, Bentovim (2000) afirma que las conductas humanas deben ser tenidas en cuenta juntamente con su contexto sociocultural, que las carencias económicas y sociales producen una transformación en las personas que pueden convertirlas en abusadoras y predispuestas a un riesgo alto y así se intentan controlar los episodios angustiantes por medio de la intimidación, en un sistema que casi no la limita, ni brinda soluciones alternativas. La violencia siempre es eficaz y certera a la hora de cumplir sus objetivos devastadores, desplegándose a la par del ser humano desde su individualidad y exponiéndolo, junto a su entorno más íntimo, a la inevitable interrelación con los otros, lo que genera que hechos producidos en la intimidad de los hogares impacten en las esferas psicosociales (Caruso, 2016). En idéntico sentido, Liu et al. (2021) explicitan sus hallazgos en términos de aumento de síntomas de depresión, mayores riesgos de suicidio y una menor satisfacción con la vida después de sus experiencias de violencia doméstica.

Entiende Perrone (2012) que la agresividad, por medio de una inhibición adaptada y funcional, regula las relaciones entre las personas, lo cual permite la viabilidad de la vida social y que lo opuesto da lugar a actos violentos. Interpreta que la agresividad es un impulso a favor de la conservación de la vida. En la observación clínica aparecen tres tipos diferentes de violencia entre los individuos, provocadas por la carga o ausencia de agresividad. En primer lugar, Perrone (2012) define como violencia agresión a la que se establece entre individuos que, ubicados en posiciones equivalentes y en una 
relación confrontante, exhiben la violencia en forma de agresión, manifestándola en forma de gritos, de intimidaciones, de agresiones verbales y físicas.

En segundo término, cuando las relaciones son complementarias, toman la forma de violencia castigo, debido a la deficiente capacidad para defenderse que presenta uno de los individuos que se ve obligado a soportar los ataques del otro sin poder oponerse. La falta de estima y el autodesprecio hacen que la persona agredida se acomode a esa realidad, sin requerir protección social alguna, ni siquiera policial o judicial, por lo que, en los casos más graves, llega a convertirse en sometimiento y dependencia (Perrone, 2012).

En tercer lugar, se identifica la violencia represalia, variante de la anterior, que aparece cuando el individuo que se encuentra en posición desfavorable, al verse obligado a sobrellevar el castigo, ofrece resistencia, a pesar de la relación negativa de fuerzas. Este tipo de violencia denota los intercambios entre el que violenta y se impone, contrapuesto al que absorbe las agresiones y las soporta, esperando revertir la situación (Perrone, 2012).

\section{Familia}

Existen familias monoparentales, ensambladas, integradas por hijos provenientes de otros vientres maternos, con integrantes de diversas preferencias sexuales, que cuentan con varias separaciones, con divorcios, nuevas uniones, con niños a cargo de abuelos, con y sin hijos. Todas ellas son familias cuyo modelo se denomina heterogeneidad familiar (Losada, 2015; Losada y Marmo, 2017, 2019).

Las relaciones de pareja se desarrollan en dos espacios que las componen de forma esencial, según aseguran Linares y Soriano (2017), refiriéndose, por un lado, a la conyugalidad y, por otro, a la parentalidad. Señalan que las dos se interrelacionan y son influenciadas por el transcurso del tiempo en un recorrido natural del ciclo vital, definiéndolas como dimensiones relacionales.

Por su parte, Gutiérrez et al. (2018) aseveran que es la familia el primer espacio para socializar, que los objetivos que alcanza como institución social se deben a que posee funciones muy específicas y a que se reflejan en ella todos los vínculos sociales en su conjunto (al igual que sus propias contradicciones), pero también a que, simultáneamente, ella es considerada un universo íntimo. Respecto de la noción constitucional de familia, Herrera (2015) asevera que la más acorde sería la clasificación amplia, que responde a una tipología flexible, sensible y adaptable rápidamente a los cambios sociales. Es ésta la adoptada por la Constitución Nacional Argentina al referirse a la protección integral de la familia.

\section{Violencia familiar}

La violencia familiar es un fenómeno psicosocial tan antiguo como la humanidad, basado en la inequidad de la pareja, en la disparidad de fuerzas de quienes se suponen 
pares, en la supremacía del hombre y en el dominio de lo masculino sobre la mujer. Estos fenómenos se postulan constructores de sociedades patriarcales que sostienen al machismo como filosofía basal de su existencia y modo sistemático de proceder.

Gutiérrez et al. (2018) sostienen que existe violencia familiar cuando los integrantes comienzan con conductas disfuncionales, las cuales generan problemas para la salud y el desarrollo de sus miembros en el seno del hogar familiar. La envergadura del fenómeno afecta a un más de un tercio de las parejas y se presenta en planos diversos como el físico, el emocional o el sexual (Inman y London, 2021).

En determinadas situaciones la agresividad natural de los seres humanos puede descontrolarse provocando una descarga denominada agresión. Cuando está dirigida contra otras personas, se la llama violencia y cuando dicha agresión se produce en el ámbito familiar, se la denomina violencia familiar o doméstica. Esta puede producirse no sólo por acciones, sino también por omisiones respecto de obligaciones emergentes de la propia relación interpersonal. Ya no subsisten espacios exentos ni áreas seguras cuando se despliega la violencia. Es tan así que ella se ha instalado en lo más hondo de la existencia humana, en los lugares más sensibles para las familias, es decir, en sus propios hogares (Caruso, 2017b).

\section{Justicia}

Los juzgados intervienen para hacer cesar los actos dañosos sobre los individuos afectados, a modo de objetivo principal de la legislación vigente y también a los fines de evitar la reiteración de su padecimiento, lo cual se materializa a través del dictado de medidas de protección. Dicha intervención de los órganos jurisdiccionales del Estado no sólo intenta la protección de las personas que resultan afectadas por los actos violentos, sino que también se emplaza en defensa del bienestar común y del orden público.

Dentro de las complejas tareas que enfrenta la justicia del fuero civil con competencia en los grupos primarios se destacan, sin lugar a duda, los procesos de violencia. El magistrado debe intervenir para el dictado de medidas de protección, previniendo el desenlace de los acontecimientos futuros, en el ámbito familiar y en el desempeño social de los individuos implicados (Caruso, 2017b).

El objeto principal de los procesos judiciales en este tipo de casos es el dictado, en un plazo perentorio, de medidas que interrumpan las situaciones de maltrato. Nuestra legislación en la materia enumera diferentes tipos de medidas de protección para las personas afectadas, que a criterio del juez interviniente pueden ser adoptadas. Estas van desde exclusiones del hogar familiar y restricciones para el acercamiento, $\mathrm{u}$ otros tipos de contacto, hasta medidas accesorias respecto de cuotas de dinero para el sostenimiento familiar y la suspensión de acuerdos vigentes relativos al 
contacto paterno filial o al cuidado de personas. Asimismo, faculta expresamente a los magistrados para adoptar cualquier tipo de medidas que considere necesarias (Caruso, 2017a, 2017b). En ese aspecto, los jueces deben tener en cuenta que es la propia ley la que les permite adoptar medidas que a su criterio sean conducentes, no sólo para que cesen las situaciones actuales, sino para que sobre ellos recae la enorme responsabilidad de evitar su repetición, y con ese propósito se debe determinar el nivel de riesgo en cada caso. En esa misma intervención judicial subyace la necesidad de prevenir la propagación de la problemática, es decir la repetición de las acciones violentas hacia otras personas (Caruso, 2017b).

La responsabilidad del magistrado abarca tres aspectos fundamentales a la hora de determinar las medidas urgentes que debe adoptar. El primero es hacer cesar en forma inmediata las situaciones de violencia que se estén produciendo, separar a los agresores de las víctimas y evitar la reiteración de los sucesos. El segundo es brindar la debida atención a las víctimas, poniendo a su disposición todos los recursos para la realización de adecuados tratamientos especializados en la problemática. El tercero es el tratamiento de los agresores, a través de terapias individuales y grupos de contención y ayuda; este, además de ser indispensable el apoyo y el incentivo para el cumplimiento de las medidas judiciales, resulta el principal factor protector de la sociedad toda, ya que por su intermedio se puede aspirar al encauzamiento de la agresividad y que las personas tratadas formen en el futuro, parejas sin características violentas (Caruso, 2017b).

\section{Objetivo}

Describir las consecuencias producidas por la intervención judicial en los casos de violencia familiar sometidos a la consideración de los jueces de familia o intervención de magistrados de otros fueros sobre la integridad psicosocial del vínculo parental.

\section{Método}

En esta investigación se llevó a cabo un estudio descriptivo y transversal, a partir de 453 encuestas, que buscó recabar información sobre la experiencia personal de los estudiantes universitarios, relativa a la incidencia de la intervención judicial en los procesos de violencia familiar. También se realizaron diez entrevistas en profundidad a magistradas que se desempeñan en la justicia especializada en la materia. Las entrevistas en profundidad buscaron describir las sapiencias de aquellos efectores del poder judicial que tienen a su cargo la toma de decisiones en casos de violencia doméstica. El análisis de datos se basó en la teoría fundamentada y buscó responder a los supuestos básicos de la presente investigación. De acuerdo con los principios éticos de la investigación, se administró, tanto en las encuestas como en las entrevistas, el consentimiento informado, constituyéndose como un instrumento en resguardo de los principios éticos de la investigación (Losada, 2014). 
El procesamiento de corte cualitativo de las entrevistas administradas se llevó adelante a través de la teoría fundamentada, inscripta en el contexto formal del interaccionismo simbólico, ubicado en el paradigma interpretativo posibilitando la comprensión social desde un espacio comunicacional. Los diseños de teoría fundamentada pueden interpretarse desde los campos sistemáticos, los diseños emergentes y los diseños constructivistas (Hernández Sampieri et al., 2014). El análisis de las entrevistas denoto las expresiones culturales y sociales a través de un proceso interpretativo, aportándose significados de los fenómenos de estudio (Herrera, 2018). El desarrollo cualitativo de la presente investigación se posiciona del tipo comprensivo aceptando lo valioso de expertos en la materia, siendo que el estudio interpretativo del contenido se plantea en el contenido que emergió de las respuestas cobrando sentido en el contexto en el que se desarrolla (Tylor y Bogdan, 1987). Según Marradi et al. (2007) el análisis de contenido posibilita una descomposición y clasificación de los elementos de investigación, en este caso las entrevistas llevadas adelante a funcionarias judiciales. En cuanto al cuestionario, se lo utilizó de modo estratégico para recabar datos empíricos. Este trabajo presentó carácter de transversal (Hernández Sampieri et al., 2014).

\section{Muestra}

En esta investigación se realizaron encuestas a 453 estudiantes universitarios de distintas universidades, tanto públicas como privadas, de la Ciudad Autónoma de Buenos Aires y del partido de Lomas de Zamora, en la provincia de Buenos Aires. Se recabaron datos relativos a la experiencia personal, familiar y social de los estudiantes universitarios, a partir de la participación de alumnos de veintitrés diferentes carreras, de disciplinas tales como psicología, abogacía, psicopedagogía, escenografía, relaciones públicas, relaciones internacionales, administración de empresas, enfermería, diseño y comunicación visual, trabajo social, economía empresarial, educación, relaciones internacionales, artes audiovisuales, ingeniería en producción agropecuaria, medicina, ingeniería civil, ciencias políticas, ingeniería industrial, tecnicatura en sonido y grabación, comunicación periodística, ciencias naturales, lengua y literatura, traductorado público, ciencias turismo y arquitectura. En tanto, en las entrevistas, la muestra se conformó por diez juezas del fuero de familia del Departamento Judicial de Lomas de Zamora y magistradas de la Ciudad Autónoma de Buenos Aires.

\section{Resultados}

\section{Encuestas}

Entre los resultados, se observa que del total de encuestas 133 (29,36\%) fueron personas de género masculino y 320 (70,64\%), de género femenino. De ese universo, $76(16,78 \%)$ informaron haber padecido en su grupo familiar alguna situación de violencia. De los 76 afectados, dieciséis fueron varones (12\% del total de encuestados) y 60 mujeres (18,75\% del total de encuestados). 
Se puede señalar que en un de 42 casos (91,30 \%) se indicó el haber padecido violencia de índole física, once varones $(24,39 \%)$ y 31 mujeres $(75,61 \%)$. Al mismo tiempo fueron $56(7,68 \%)$ quienes expresaron haber padecido violencia verbal, ocho varones $(14,29 \%)$ y 48 mujeres (85,71\%). En ese sentido, se registraron cinco testimonios de personas que dicen haber sufrido abuso sexual $(6,57 \%)$, un varón y cuatro mujeres. En 25 casos $(32,89 \%)$ recuerdan haber sido testigos de violencia familiar cuando eran niños, de ellos, cinco varones (20\%) y 20 mujeres (80 \%). Compulsada la base de datos se encontraron seis casos $(1,32 \%)$ con existencia de intervención judicial, en un varón $(16,67 \%)$ y en cinco mujeres $(83,33 \%)$.

De modo similar, los resultados reflejan que frente al total de las personas que integraron familias en las que existieron situaciones de violencia, siete $(9,21 \%)$ revelaron que por orden judicial algún miembro de la familia sufrió medidas restrictivas, dos personas $(2,63 \%)$ fueron impedidas de contactarse y cinco $(6,58 \%)$ señalan que además debieron abandonar el hogar. Similarmente, en términos de reconocimiento de los efectos de las medidas judiciales, el número de encuestados que reconoció el empeoramiento del contacto paterno filial, en los casos con intervención judicial, asciende a 91 (20,08\%). Se verificaron 21 casos $(4,63 \%$ de los encuestados totales, que representan el $27,63 \%$ de quienes manifestaron haber padecido violencia en su familia de origen) en los que indican que se produjo el alejamiento definitivo de alguno de los miembros.

Dentro de los estudiantes partícipes, $218(48,12 \%)$ expresaron que tomaron conocimiento de la existencia de uno o más casos de violencia familiar en su entorno informando 439 casos, es decir en varios pudieron referir a más de un caso. 109 encuestados $(24,06 \%)$ expresaron que el grupo familiar afectado ha recibido algún tipo de asistencia. De las 453 encuestas, se incluyen en esta investigación las correspondientes a alumnos de nivel universitario de quince diferentes carreras; son 195 de psicología, donde se registraron 36 casos situaciones de violencia en sus familias $(18,46 \%)$ y se manifestó, en 98 de ellos, conocer situaciones de violencia en familias de su entorno $(50,25 \%$ del total de encuestados en esa carrera).

En tanto, los alumnos de administración de empresas que totalizaron 88, expresaron, en nueve casos $(10,22 \%)$ haber padecido violencia doméstica en el seno de sus familias y 36 (40,90\% del total) manifestaron conocer casos de ese tipo de violencia padecida por personas de su entorno social. De la carrera de abogacía fueron encuestados 53 alumnos, de los cuales trece $(24,52 \%)$ reconocieron la existencia de hechos de violencia intrafamiliar en su propio entorno y $32(60,37 \%)$ sugirieron tener conocimiento de situaciones así en personas cercanas.

Las encuestas realizadas a los alumnos de la carrera de medicina fueron siete, pero ninguno de ellos expresó que en el seno de sus familias existieran situaciones de violencia, no obstante uno informó conocer situaciones así en personas de su entorno social. Integran esta investigación 27 encuestas realizadas a estudiantes de la carrera de ingeniería; la totalidad de ellos desconoció la existencia de situaciones de violencia doméstica en sus familias, sin embargo, ocho (29,63\%) indicaron conocer 
personas con familias que la padecen.

Antagónicamente, los siete alumnos de ciencias políticas encuestados no expresaron haber vivido con familias con presencia de violencia. No obstante, dos de ellos manifestaron conocer situaciones de violencia familiar en su entorno social. Fueron encuestados once alumnos de la licenciatura en enfermería, de los cuales cuatro $(36,36 \%)$ reconocieron la presencia de situaciones de violencia en sus propias familias $\mathrm{y}$ ascienden a seis $(54,55 \%)$ los casos de quienes dicen haber tomado conocimiento de ese tipo de circunstancias en familias de personas allegadas o conocidas. Los alumnos de la licenciatura en relaciones públicas que rellenaron las encuestas alcanzan un total de diecisiete, de los cuales tres (17,64\%) vivieron situaciones de violencia intrafamiliar, mientras que diez $(58,82 \%)$ señalaron ese tipo de escenarios en familias de su ambiente social.

Sin embargo, en la licenciatura en relaciones internacionales, se obtuvo la participación de cinco alumnos de los que fueron dos quienes manifestaron la presencia de violencia en sus familias y uno registró situaciones análogas en otras familias de su conocimiento. A los cuatro alumnos de comunicación periodística que se encuestaron no indicaron casos de violencia en sus familias, pero tres de ellos (75\%) informaron conocer casos en familias de su entorno social.

La investigación incluye quince encuestas completadas por los alumnos de artes audiovisuales y tres de ellos (20\%) fueron quienes reconocieron padecer violencia familiar, y nueve (60\%) manifestaron conocer esas circunstancias en familias conocidas. En tanto, los tres alumnos encuestados de la licenciatura en turismo respondieron negativamente a la presencia de violencia en sus familias y en las de su entorno.

De la carrera de Contador Público seis respondieron: un caso $(16,66 \%)$ con presencia de violencia familiar en su propia estirpe y dos $(33,33 \%)$ con conocimiento de casos de esas características en otras familias. En economía fueron cuatro los alumnos que completaron la encuesta y, como resultado, se obtuvo que uno de ellos (25\%) había padecido situaciones de violencia familiar. Tres alumnos (75\%) reconocieron la existencia de situaciones de esa índole, en otras personas de su entorno social. Con respecto a las siete las encuestas completadas por alumnos de educación y profesorados universitarios, dos de ellos $(28,57 \%)$ manifestaron haber vivido situaciones de violencia familiar y cuatro de ellos $(57,14 \%)$ tomaron conocimiento de la existencia de situaciones de violencia en personas cercanas. Se encuestó a estudiantes de tres carreras cuya muestra no resulta significativa: un alumno de trabajo social, dos de traductorado público y uno de psicopedagogía, es decir, cuatro encuestados. No obstante, dos de ellos (50\%) informaron haber padecido situaciones de violencia doméstica en su grupo familiar y tres $(75 \%)$ del total manifestó tener conocimiento de situaciones semejantes en otras familias de su entorno social.

\section{Entrevistas}

En relación con el análisis cualitativo del impacto de las medidas judiciales en la integridad familiar, se desarrollaron diez entrevistas en profundidad a magistradas 
que se desempeñan en la justicia especializada en la materia, con competencia en violencia familiar, para recabar sus saberes y conocimientos. Bajo la pretensión de profundización sin buscar una generalización de los resultados se describieron los resultados agrupándolo por ejes según los rasgos particulares que permitieron cualificar los hallazgos (Herrera, 2018). Como corolario de dichas entrevistas, la información obtenida se categorizó en los siguientes ejes.

Proporción de las resoluciones judiciales dictadas en procesos de violencia familiar se adecúan a lo dispuesto en la legislación vigente. Las entrevistadas coincidieron en señalar que entre el 70 y el $90 \%$ de dichas resoluciones se ajustan a las normas disponibles en la materia. Aclararon que, de no ser así, se estaría contra derecho. Con respecto a las causas que no se ajustan a la legislación imperante, las participantes lo justificaron en la falta de presentación de las víctimas ante los juzgados atribuyéndoselo a una carencia de información en la sede donde radicó la denuncia. También, debido a que la urgencia no permite adecuarse totalmente a la teoría legislativa, o en virtud de que en los casos de escasa gravedad no siempre se cumplen los plazos. Se indicó además que los magistrados interpretan de modo diverso la normativa legal, por ende, a veces se apartan de la letra de la ley para resolver según su opinión. Otra mirada fue que, en general, no se les otorgan medidas en casos de denuncias mutuas, o ante la reiteración de denuncias, o debido a la falta de asesoramiento jurídico de las personas afectadas. Se justifican los incumplimientos fundamentalmente en los plazos legales o en los casos en que existen dificultades para notificar a la persona denunciada, o inconcurrencia de la persona afectada. Por último, marcan que la ley no prevé todas las situaciones que se presentan en la realidad, lo cual justificaría las resoluciones desajustadas.

Modo en que se estima que impactan psicológica y socialmente las medidas de protección que ordena la justicia en los procesos de violencia familiar. Las entrevistadas explicaron que concientiza a las partes, si es que las medidas se efectivizan, para lo cual es fundamental el abordaje terapéutico para victimario y para víctima. Aseguraron que impactan en forma directa, porque el denunciante se retira conforme al obtener las medidas, que el impacto es positivo y que la sociedad también lo recibe positivamente. Otra de los participantes ponderó que, en general, no se percibe que las partes presten debida importancia a las resoluciones judiciales. Usualmente, según otra entrevistada, impactan de forma positiva debido a que tienen un efecto reparatorio y que la medida de protección se vuelve una minimización del daño. Sostuvo además que hay muchas variables y que, en ocasiones, los hijos culpan a las madres por la exclusión del padre. Aseveraron que las medidas judiciales vienen para hacer cesar la violencia, la cual en algunos casos no se reitera y en otros sí, y que las mujeres terminan socioeconómicamente perjudicadas, pero emocionalmente empoderadas. Calificaron las medidas dispuestas por la justicia como positivas, debido a que marcan un hito en la impronta familiar a modo de ley inscripta desde el exterior, porque es una forma de organizar el caos y la disfuncionalidad familiar. Sostuvieron que la persona denunciante se siente protegida, en virtud de que la 
medida judicial es una herramienta de protección, y aclararon que el denunciado tiene diversas reacciones, según cada caso en particular. En cuanto a lo psicológico hay un quiebre en las relaciones familiares y socialmente se percibe como un logro el accionar judicial. Explicaron que, en realidad, las familias no acompañan lo suficiente, que lo hacen en un primer momento y luego, no. Consideraron a las medidas judiciales en forma positiva, no obstante podrían impactar en forma negativa si no hubiera acompañamiento del poder ejecutivo y si en sede penal no se efectivizaran las sanciones por incumplimiento de dichas medidas judiciales, es por ello por lo que entonces hay personas que descreen del funcionamiento de la justicia.

Modo en el que interpretan que inciden en la continuidad de la relación parental la aplicación de la legislación vigente en materia de violencia familiar. Las profesionales señalaron que, generalmente, con el victimario la relación se obstaculiza; en el 80\% de los casos, por no trabajarse con él, se interrumpe la relación. Sostuvieron que dificulta la continuidad de la relación la gestión del conflicto, modificando la relación de padres e hijos y que el vínculo paterno filial a veces agota la medida. Además, aseveraron que en realidad el contacto se obstaculiza y que incide sustancialmente la no vinculación con terceros para mantener la relación paternofilial. En este sentido, explicaron que es una problemática muy compleja y que, por no estar contemplada en la legislación, los tribunales suelen improvisar y la continuidad vincular deteriora. Por un lado, expusieron que, en general, el hombre violento se aísla de sus hijos porque no puede diferenciar entre el conflicto de la pareja y la relación filial. Por otro, que, en algunos casos, hay división de núcleos fraternos, porque se deciden, de forma manipuladora, separar a los hermanos. Una de las funcionarias entrevistadas sostuvo que se establecen necesariamente, y en unas ocasiones, alianzas entre los miembros y, en otras, coaliciones entre otros. Por medio de terapias todo se puede trabajar, afirmó una entrevistada, quien además explicó que ante una persona violenta suele necesitarse la revinculación con los hijos luego de un tiempo. Para ampliar lo anterior, otra expresó que incide negativamente y que las consecuencias son difíciles de revertir, dado que representa un quiebre muy importante en los lazos afectivos. Sin embargo, otra de las personas entrevistadas expresó que no continúan iguales los lazos familiares, que seguramente habría un discurso peyorativo de la persona denunciada. Por último, afirmaron que incide negativamente la aplicación de la legislación vigente en materia de violencia familiar y que por las mismas razones no se logra una revinculación adecuada, sana, no patológica.

Relación existente entre la magnitud de la violencia padecida por las personas afectadas y la dimensión de las medidas de protección dictadas judicialmente. Las entrevistadas afirmaron que se intenta que exista proporcionalidad para lograr que a mayor gravedad, mayor severidad de la medida, lo cual queda supeditado al riesgo que el equipo técnico haya determinado. Una de ellas destacó que sería proporcional a la gravedad de los hechos de violencia, siempre dentro del límite que la legislación impone, debido a que no existe una medida de protección para cada caso. Surge de las entrevistas un fenómeno que se reitera en los casos 
de violencia familiar judicializados y refiere a que las medidas son mayores que la gravedad que revisten, esto debido a la no existencia de medidas de menor impacto. Una de las juezas sostuvo que la idea es que las resoluciones tiendan a corresponderse con el maltrato y que el impacto de dicho maltrato es íntimo y personal, no ligándose solamente al ámbito judicial. De forma similar, se señaló que las medidas judiciales no tienen dimensión, que la única variable es el tiempo, por lo que no hay concordancia entre la medida adoptada y la magnitud del maltrato sufrido. Una de las participantes explicitó que en ocasiones no tiene relación, dado que se han observado medidas extremas en casos de riesgo bajo y por el contrario, en otras situaciones con riesgo altísimo las medidas otorgadas no alcanzan, o resultan insuficientes, y que las resoluciones no son proporcionales; que en algunos casos no son suficientes las medidas que la justicia puede dictar ya que en ocasiones se observa que son muy leves en casos con riesgo alto, a pesar de ser las más altas previstas, y que a veces no existe la posibilidad de proteger totalmente a las víctimas. En otra respuesta se indicó que muchas veces es mayor la violencia sufrida que la fuerza de protección de la medida dictada, que en otras ocasiones es desproporcionada la medida adoptada ante un hecho leve, dado que no existen determinaciones mínimas que no afecten en alguna forma la libertad personal de la persona denunciada, una vez que accedió a la justicia, y que muchas veces las disposiciones judiciales no se cumplen porque las mismas personas que realizan las denuncias no pueden sostener las medidas que solicitan, ya sea por razones económicas, psicológicas o falta de lugares de tratamiento especializado que acompañe. Diversas autoridades judiciales entrevistadas destacaron que las medidas son generales y que no cambian para cada caso, lo que las hace acciones estancas y de aplicación subjetiva, ya que dependen de la interpretación y decisión del juez. Otra funcionaria afirmó que son totalmente desparejas, que hay medidas insuficientes, las que hoy se encuentran disponibles y no provocan el efecto deseado debido a innumerables factores, especialmente de índole económica, o social y por la falta de acompañamiento de la persona afectada.

Estimación respecto de las resoluciones judiciales de los casos en procesos de violencia familiar, que contienen determinaciones que establecen la exclusión de alguno de los integrantes del sistema familiar. Una de las entrevistadas expuso que en el 10\% de los casos las medidas adoptadas son las de exclusión del hogar, pero si se toman en consideración las que implican exclusión del contacto de algún integrante, asciende al $90 \%$. Por un lado, se indicó que las exclusiones o impedimentos de contacto son, aproximadamente, el $10 \%$, no obstante casi todas las medidas son restrictivas del contacto de alguno de los integrantes de la familia, alcanzando el 85 a 90\%. A su vez, una de las personas entrevistadas señaló que las exclusiones del hogar son las de menor cantidad, aproximadamente el $20 \%$, pero el contacto se pierde en casi todos los casos con alguno de los familiares, en un $90 \%$. Por otro lado, una de las profesionales aseveró que las exclusiones del hogar no sobrepasarían el $10 \%$ de los casos, pero que al referirse a los distanciamientos de contacto entre las partes, el número es muy alto, entre el $80 \%$ y el $90 \%$ de los casos. En este sentido, una de las entrevistadas señaló que en todos los casos en que se toman acciones judiciales 
hay alguien excluido, dado que de eso se tratan las resoluciones, es decir, sacar a algún miembro del contacto para hacer cesar la violencia. Una de las funcionarias judiciales partícipes reveló que en el $70 \%$ de los casos se produce el alejamiento de alguno de los integrantes de la familia. En este sentido, en otra entrevista se destacó que alcanzan un $25 \%$ las exclusiones del hogar e impedimentos de contacto, pero que en referencia a la restricción del contacto de denunciados no convivientes, se llega prácticamente al total, en un $95 \%$. Por su parte, otra entrevistada destacó que un 30\% de las situaciones son medidas de alejamiento del hogar, el resto posee otras características que también suponen el no contacto con alguno de los familiares, un total aproximado del 90\%. Señalaron también que las disposiciones judiciales en estos procesos son transitorias, lo cual queda demostrado con el alto índice de reincidencia en cuanto a la producción de hechos similares por parte de las personas denunciadas. En el período de vigencia de las órdenes de exclusión del contacto es prácticamente total, un $95 \%$.

Consideración en que la intervención judicial, en procesos de violencia familiar, modifica los vínculos familiares cuando se produce la exclusión de alguno de los miembros o la disminución de la comunicación entre ellos. Las entrevistadas expresaron que incidirá en la medida que se trabaje con las partes, porque si ellos no hacen terapia el resultado es nulo. Una de ellas manifestó que impactan en la continuidad del vínculo siempre. En este sentido, otra planteó que los hijos generalmente ven entorpecido el vínculo con su padre, debido a la inexistencia de terceros para que faciliten el contacto o por la voluntad de la denunciante. Es una dinámica donde todo es relativo y es caso por caso, y existe una modificación temporaria, pero a nivel emocional no se modifica el vínculo; además, se explicó que de no mediar tratamiento, la intervención judicial incide en forma negativa sobre los vínculos familiares. Se señaló también que no afectan en forma definitiva, dado que son medidas cautelares de aplicación momentánea, mientras que durante la vigencia de la esta la incidencia es negativa. Se sostuvo que incide negativamente, porque hay un alejamiento, una imposibilidad de diálogo y, en la mayoría de los casos, no existen otros referentes familiares que aporten soluciones. En contraposición, una de las autoridades entrevistadas explicó que no considera que se produzca definitivamente el distanciamiento familiar porque las medidas son transitorias y que luego los vínculos son disfuncionales; que no es la intervención, sino las acciones en general, las que socavan el vínculo. Para finalizar, una entrevistada expresó que siempre hay un cambio en ese núcleo de familia; a partir de ello queda la familia separada y los hijos en el medio.

Propuestas de modificación de fondo en la legislación vigente en materia de violencia familiar. Para las profesionales, los procesos de violencia se debieran tramitar en juzgados especializados y además sería esperable realizar acciones en educación y en salud en el ámbito municipal de desarrollo social. Los procesos de violencia familiar deberían iniciarse en el sistema de salud; las causas de violencia familiar deberían iniciarse en organismos intermedios no judiciales que permitan 
una primera intervención de distintos profesionales, a fin de evaluar las situaciones planteadas y, así, seleccionar las correspondientes a la materia para su tratamiento y derivación a la justicia, en caso de corresponder. Las variables del acoso no están suficientemente legisladas, al igual que el hostigamiento en sus diversas formas. A los fines de prevenir perjuicios mayores, deberían existir tanto una intervención de la justicia penal, como medidas de coerción conjuntas, es decir que la respuesta judicial sea integral. De igual modo se plantea la necesidad de crear juzgados especializados con capacitación profunda para todo el Poder Judicial en perspectiva de género. Se comprende también que un fuero especial beneficiaría la escucha de las personas afectadas y la dinámica del proceso. En los aspectos penales debería trabajarse con el condenado, realizando un diagnóstico previo de interacción familiar por un equipo interdisciplinario, integrado por psicólogo, psiquiatra y trabajadora social. Esto enmarcado dentro de las políticas públicas acerca de la problemática de la violencia familiar y que estén aceitados los mecanismos entre a justicia civil y la penal. Por último, analizar cada artículo de la legislación vigente para determinar su efectividad en cuanto a la aplicación. Reformar la ley basándose en la realidad de la sociedad. De las entrevistas a funcionarias judiciales se concluyó que las medidas judiciales impactan siempre en la continuidad del vínculo familiar y de igual modo indicaron que los hijos, generalmente, ven entorpecido el vínculo con su padre y aseveraron que, posteriormente, los vínculos son disfuncionales. Las acciones en general socavan el vínculo y en todos los casos hay una modificación en ese núcleo de familia y, a partir de ello, la familia queda separada y los hijos en medio del conflicto.

\section{Discusión}

La violencia doméstica trasciende la vida y los intereses de las familias a las que traspasa para alojarse en el seno de la sociedad. Desde allí se expresa en diversas formas y estadios y adopta características propias en cada ámbito en el que le toca desarrollarse. La violencia siempre es eficaz y certera a la hora de cumplir sus objetivos devastadores; se despliega a la par del ser humano desde su individualidad y lo expone, junto a su entorno más íntimo, a la inevitable interrelación con los otros en el interior de los demás senos de la comunidad. Esto genera que hechos producidos en la intimidad de los hogares, impacten en las esferas psicosociales, para cumplir con su firme propósito de propagación social.

El trabajo de campo de la presente investigación con estudiantes universitarios recaba que, del total de los 76 individuos que integraron familias en las que coexistían situaciones de violencia, siete de ellas $(9,21 \%)$ revelaron que por orden judicial algún miembro de la familia sufrió medidas restrictivas; dos de ellos apuntaron que dos personas fueron impedidas de contactarse con integrantes de sus familias y en cinco casos advirtieron sobre la existencia de individuos que además debieron abandonar el hogar familiar. El número de sujetos encuestados que reconocen el empeoramiento del contacto paterno filial en los casos con intervención judicial asciende a $91(20,08 \%)$. Se han verificado 21 casos $(27,63 \%)$, de quienes manifestaron 
haber padecido violencia en su familia de origen, en los que indican que se produjo el alejamiento definitivo de algún miembro de la familia.

La incidencia de los aprendizajes sociales respecto de las conductas violentas, llevadas a cabo con las personas del entorno familiar, podría aceptarse como factores culturales condicionantes para los buenos o malos tratos en el seno de la familia. Las culturas patriarcales con su emergente necesario, el machismo, se encuentran presentes en la mayoría de los casos de agresiones intrafamiliares. Con respecto a la integridad psicosocial del vínculo parental, se pudo determinar, a través de la presente investigación, que en los casos de violencia familiar sometidos a la consideración de los jueces con competencia en el área, o aún más específicamente en denuncias de violencia familiar, se producen diversas consecuencias a partir de las resoluciones emanadas de dicha intervención judicial.

Los saberes de Arendt (2005), Bentovim, (2000), Herrera (2015), Inman y London (2021), Losada (2015), Perrone (2012) y Sanmartín (2004) expresan la problemática de la violencia en sus distintas esferas, ya sea política, social o familiar. Se refieren a los sujetos agresivos y profundizan en la violencia familiar, determinando tanto las consecuencias de la disparidad en la relación entre agresividad y vulnerabilidad, como en los distintos factores que las producen y fomentan. Esto permite visibilizar un fenómeno que, asociado a ella, aparece invariablemente como generador, consecuencia o complemento, pero siempre necesario e infaltable, como la relación de poder, que se manifiesta muy especialmente en la intimidad de las familias.

Esa violencia aprendida en la intimidad del hogar parental, o desarrollada y practicada en el suyo propio, necesitó de la supremacía de uno sobre los otros y de la dominación de quien ostentara el poder en quienes lo padecieran, lo que establece una ventaja del más fuerte sobre los más débiles; esta es transferida hacia el exterior, en la medida que puedan establecerse otras relaciones de poder en las que estos sujetos sean los más fuertes, por ende dominadores o, por qué no, dado su grado de vulnerabilidad, también los sometidos.

Pudo constatarse que las sentencias judiciales en procesos de violencia familiar contienen determinaciones que responden a lo preceptuado por la legislación vigente con la consecuente exclusión de uno o más integrantes del sistema familiar. A partir de ello, se logró determinar que las resoluciones judiciales adoptadas en el marco de los procesos de violencia familiar son jurídicamente aceptables y se encuentran enmarcadas en la legislación vigente, tal como lo manifiestan en las entrevistas realizadas a las funcionarias del Poder Judicial que exteriorizaron que lo hacen en un alto porcentaje y que, de no ser así, se estaría en contra del derecho. Se destacó que en los casos donde las disposiciones no logran dar estricto cumplimiento a la ley esto se debería a razones externas a los juzgados, como la falta de presentación de las víctimas ante los tribunales por no habérsela informado correctamente en el organismo donde radicó la denuncia. Asimismo, se reveló que pueden registrarse inexactitudes acerca de la teoría legislativa, debido a la urgencia de algunos casos, como también puede observarse algún retraso en el cumplimento de los plazos en las 
situaciones de escasa gravedad. Los casos de incumplimientos en los que se observan fundamentalmente ligados de los plazos legales, o en donde existen dificultades para notificar a la persona denunciada, o por inconcurrencia de la persona afectada a la sede judicial, se presentan a modo de justificaciones y no permiten la resolución del conflicto. Se subraya que la ley no prevé todas las situaciones que se presentan en la realidad, con lo cual se explicarían las resoluciones desajustadas que en ocasiones deben arrogar los magistrados.

La investigación dio cuenta que las medidas judiciales adoptadas en los casos de violencia familiar no resultan optimas para la integridad psicosocial de la familia, ya que las acciones que realmente se efectivizan persiguen el objeto de concientizar a las partes; para lograrlo es fundamental el abordaje terapéutico, tanto para la persona denunciada como para la afectada. En idéntico sentido resultó concluyente que las medidas adoptadas de impacto directo, en las cuales la persona denunciante se retira conforme al obtener las restricciones pretendidas, generan una señal positiva también en la sociedad. El impacto efectivo de las disposiciones que dicta la justicia sería debido a su efecto reparatorio.

Otro de los hallazgos de la presente investigación da cuenta de que en ocasiones los hijos culpan a las madres por la exclusión del padre. A la vez se expone entre las conclusiones que las medidas judiciales llegarían para hacer cesar la violencia, aunque en algunos casos no se reitera pero en otros sí y las mujeres terminan socioeconómicamente perjudicadas, pero emocionalmente empoderadas. Se constituye como un aporte de la investigación la consideración de jueces y de autoridades judiciales de las medidas dispuestas como positivas, debido a que marcan un hito en la impronta familiar a modo de ley inscripta desde el exterior, como forma de organizar el caos y la disfuncionalidad familiar. La persona denunciante se siente protegida, transformándose la medida judicial en una herramienta de protección. En el ámbito de la psicología de la familia se produce un quiebre en las relaciones y socialmente se percibe como un logro el accionar judicial. Las medidas judiciales podrían impactar en forma negativa si no hay acompañamiento del poder ejecutivo y si en sede penal no se efectivizan las sanciones por incumplimiento de dichas medidas.

La problemática de la sociedad moderna, al igual que como sucedió con las anteriores, es que demostró su propia incapacidad individual y colectiva para generar las condiciones necesarias en las que el ser humano pueda vivir y desarrollarse disfrutando su tierra, de su lugar, de su espacio y de su cobijo, sin tener que padecer las consecuencias de la violencia generada por él. Tanto es así que no sólo la sufrió en esos tiempos en los que su vida transcurría a la intemperie rodeado de agentes externos agresivos y ante la total carencia de factores protectores, sino que la soporta posteriormente en los rincones más íntimos de su guarida, de su choza, de su refugio, de su hogar. La violencia familiar como fenómeno psicosocial, por ende exclusivo de la raza humana, existió desde que se tiene registro de la vida de mujeres y de hombres y resulta evidente que no supieron, no pudieron, o no quisieron, detenerla, 
ni siquiera morigerarla. Así llegó hasta nuestros días en evidente relación entre individuos y sociedad, conviviendo cotidianamente con la violencia en el ámbito de sus familias. Por esto es que el hombre y la mujer no supieron sacar de su seno, o al menos dejar fuera de su vida, esa actitud dañina que desde siempre los lastima, los enferma, les envejece y muchas veces los mata, habiendo transformado, a través de los siglos, la agresividad necesaria para afrontar su vida y lograr la subsistencia, en una característica esencial de su ser que los destruye, la violencia familiar.

La violencia doméstica, pequeña, casi insignificante en cuanto a su poder de fuego, pero enorme en cuanto a su dimensión y a su duración, no pudo ser eliminada y ni siquiera disminuida por el hombre de hoy, lo cual se considera un gran fracaso de la humanidad que logró descubrir tanto el átomo, como el cosmos, pero no los mecanismos por los cuales acabarla (Caruso, 2017b). No es esperable, según Caruso (2016), que una sola persona, refiriéndose a la afectada por los hechos de violencia, pueda cumplir eficientemente con tantas responsabilidades, como las generadas a partir de su denuncia, por lo que se podría concluir que la solución obtenida a partir de la intervención judicial, lejos de ponerla a salvo de las terribles consecuencias de la violencia, puede llegar a exponerla de manera frontal con el perfil más agresivo y a veces letal de su agresor una vez denunciado.

El régimen legal vigente en materia de violencia familiar no permite, luego de su aplicación en el ámbito judicial, la continuidad de la relación de los padres con hijas e hijos, lo que da como resultado que con el victimario la relación se obstaculice por no trabajarse el vínculo con él. Se dificulta la continuidad de la relación de la gestión del conflicto, lo que modifica la relación entre padres e hijos y que a veces agota el vínculo paterno-filial. El contacto se obstaculiza, incidiendo sustancialmente la no vinculación con terceros, ya sea familiares o no, para mantener el vínculo paterno filial. Se trata de una problemática compleja y que por no estar contemplada en la legislación, los tribunales suelen improvisar, por lo que la continuidad del vínculo se deteriora.

El hombre violento se aísla del vínculo porque no puede diferenciar entre el conflicto de pareja y la relación filial. En algunos casos hay división de núcleos fraternos porque se decide en forma manipuladora separar a los hermanos. Se establecen necesariamente y en ocasiones, alianzas saludables entre los miembros y, en otras, coaliciones. Por medio de terapias todo se puede trabajar, las funcionarias afirman y además explican que ante una persona violenta suele necesitarse la revinculación con los hijos luego de un tiempo. Los hechos de violencia en el ámbito de la familia inciden negativamente y las consecuencias son difíciles de revertir, dado que representan un quiebre muy importante en los lazos afectivos, siendo que no persisten iguales los vínculos familiares y que seguramente habrá un discurso peyorativo de la persona denunciada. Por lo expuesto, la aplicación de la legislación vigente en materia de violencia familiar no resulta asertiva a los fines de revinculación sana no patológica.

Caruso (2016) afirma que no se puede predecir el comportamiento de un hombre violento cuando está acorralado, ni asegurar cómo reaccionará al saberse descubierto y expuesto en sus más graves manifestaciones, lo que torna impredecible el desenlace 
de su vida al divisar que perdió el control sobre la persona que cree y siente suya. Tanto las leyes de protección, como las medidas que ellas disponen, aplicadas con los recursos y procedimientos actuales, lejos de proteger a las víctimas, las exponen peligrosamente ante personas violentas que no sólo hacen oídos sordos a las negativas de las primeras, sino que desconocen toda autoridad que se interponga ante su propia convicción (Caruso, 2016).

Las acciones judiciales adoptadas que implican la exclusión del hogar son las de menor cantidad o el impedimento de contactarse de la persona denunciada. Esto ocurre si se toman en consideración las medidas que impliquen la exclusión del contacto entre integrantes de la familia, ya que el contacto se pierde en casi todos los casos con alguno de los familiares dado que todas las medidas son restrictivas de la comunicación de alguno de los integrantes de la familia. En el período de vigencia de las medidas, la exclusión del contacto es prácticamente total y en los hechos es el $100 \%$ porque siempre algún miembro es excluido, o se autoexcluye.

En la justicia se intenta lograr proporcionalidad para que, a mayor gravedad del hecho de violencia, exista mayor severidad de la sanción, lo cual queda supeditado al riesgo que el equipo técnico haya determinado, siempre dentro del límite que la legislación impone, debido a que no existe una acción de protección específica para cada caso. En una gran proporción, las medidas de protección son mayores que la gravedad que revisten debido a la no existencia de otras de menor impacto. La idea es que las resoluciones tiendan a corresponderse con el maltrato, que es íntimo y personal, por lo cual excede al ámbito judicial. Las intervenciones judiciales no tienen dimensión propia, dado que la única variable posible es el tiempo de su aplicación, que en ocasiones no tiene relación, por lo que no siempre hay concordancia entre la medida adoptada y la magnitud del maltrato sufrido. Se han observado acciones judiciales extremas en casos de riesgo bajo y, por el contrario, en otras situaciones con riesgo altísimo las resoluciones judiciales otorgadas no alcanzan, o resultan insuficientes. Estas no son proporcionales porque en algunos casos no son suficientes las acciones que la justicia puede dictar.

En ocasiones, se observan dictámenes muy leves, a pesar de ser las más altas previstas, en casos con riesgo alto y en ocasiones no existe la posibilidad de proteger totalmente a las víctimas. Muchas veces, es mayor la violencia sufrida que la fuerza de protección impuesta por los magistrados y, en otras ocasiones, es desproporcionada ante un hecho leve, dado que no existen resoluciones mínimas que no afecten de algún modo la libertad de la persona denunciada, una vez que se accedió a la justicia. En determinadas circunstancias las disposiciones judiciales no se cumplen porque las mismas personas que realizan las denuncias no pueden sostener las acciones protectivas que han solicitado por razones económicas, psicológicas o falta de lugares de tratamiento especializado que las acompañen y contengan.

En línea con el pensamiento de Horton (1986) y de Laurens (2006), se concluye que la desintegración familiar es uno de los inconvenientes con mayor ocurrencia en la sociedad, es el factor que provoca la pérdida de la unidad de la familia y representa 
además el componente que en mayor medida tensiona emocionalmente a los niños.

En concordancia con Caruso (2016), Dutton y Golant (1997), Echeburúa (citado en Sanmartín, 2004), Lamberti (2016) y Payarola (2016), se determina que resulta imperiosa la necesidad de brindar asistencia especializada a los agresores domésticos, dado que la intervención judicial no resulta exitosa por el solo hecho de haber provocado la separación de la pareja, sino que es responsabilidad del juez interviniente evitar la reiteración de las conductas del agresor intrafamiliar con otra pareja y por ende con otro grupo familiar. No es de esperar que espontáneamente el maltratador revea sus conductas y las mejore por el bien de su familia actual, la futura y la sociedad toda.

Las disposiciones judiciales interpuestas para hacer cesar los actos dañosos sobre las personas afectadas -objetivo principal de la legislación vigente-, a través de las medidas que disponen para su protección y en pos de evitar la reiteración de sus padecimientos, ocasionan efectos colaterales inevitables respecto del contacto entre la persona sancionada por la justicia y sus hijas o hijos. Esto ocurre ya que al excluirlo del hogar familiar, o impedirle el acercamiento a las personas afectadas en busca de la debida protección que la justicia debe brindar, se evita también la normal continuidad de la relación afectiva, en ocasiones temporariamente, en otras por largos períodos de tiempo y en algunos casos reviste carácter de definitivo, lo que provoca la desintegración familiar de manera no deseada.

\section{Referencias}

Arendt, A. (2005). Sobre la violencia. Alianza.

Bandura, A. y Walters, R. H. (1987). Aprendizaje social y desarrollo de la personalidad. Alianza Editorial S.A.

Bentovim, A. (2000). Sistemas organizados por traumas: el abuso físico y sexual en las familias. Paidós.

Caruso, J. M. (2016). El femicidio en la violencia de género denunciada. Revista Borromeo, 7, 37-45. HTTP://BORROMEO.KENNEDY.EDU.AR/ ArticulosNuevos/Caruso7.PDF

Caruso, J. M. (2017a). Familia, justicia y venganza. Actualidad Psicológica, XLII (468), 13-15.

Caruso, J. M. (2017b). El agresor intrafamiliar frente a la justicia civil. Revista Jurídica Universidad Nacional de Lomas de Zamora, 4, 1-17. HTTP:// REPOSITORIO.UNLZ.EDU.AR:8080/BITSTREAM/ HANDLE/ $123456789 / 267$
Dutton, D. G. y Golant, S. K. (1997). El golpeador. Un perfil psicológico. Paidós.

Gutiérrez, G. M. A., Gómez, C. L. M., Benítez, H. P., Valdés, A. M. B., Pérez, R. P. G. y Paz, L. G. (2018). Estrategia de promoción de salud frente al maltrato infantil en la familia. Mediciego, 24(3), 33-52. HTTP://WWW.REVMEDICIEGO.SLD.CU/ INDEX.PHP/MEDICIEGO/ARTICLE/VIEW/930/1391

Hernández Sampieri, R. Fernández Collado, C. y Baptista Lucio, P. (2014). Metodología de la Investigación. Editorial McGraw Hill.

Herrera, M. (2015). Manual de derecho de las familias. AbeledoPerrot.

Herrera, C. D. (2018). Investigación cualitativa y análisis de contenido temático. Orientación intelectual de revista Universum. Revista General de Información y Documentación, 28(1), 119.

Horton, P. B. (1986). Sociología. McGraw-Hill. 
Inman, E. M., y London, B. (2021). Self-silencing mediates the relationship between rejection sensitivity and intimate partner violence. Journal of Interpersonal Violence, 36 (3-4) 886-908.

Lamberti, S. (2016). Violencia masculina intrafamiliar. Una visión integradora desde el psicoanálisis y el derecho. 20XII Grupo Editorial.

Laurens, Y. (2006). Desintegración familiar. Sucre.

Linares, J. L. y Soriano, J. A. (2017). Pasos para una psicopatología relacional. Revista Mexicana de Investigación en Psicología, 5(2), 119-146.

Liu, M., Xue, J., Zhao, N., Wang, X., Jiao, D., y Zhu, T. (2021). Using social media to explore the consequences of domestic violence on mental health. Journal of Interpersonal Violence, 36(34), 1965-1985.

Losada, A. V. (2014). Uso en investigación y psicoterapia del consentimiento informado. En B. Kerman y M. R. Ceberio (comps.), En búsqueda de las ciencias de la mente. Investigación en psicología sistémica, cognitiva y neurocientífica (pp. 159-167). Ediciones Universidad de Flores.

Losada, A.V. (2015). Familia y psicología. Dunken.
Losada, A. V. y Marmo, J. (2017). Hacia la comprensión de los procesos familiares. Dunken.

Losada, A. V. y Marmo, J. (2019). Manual de psicología de la familia. EDUCA.

Marradi, A., Archenti, N., y Piovani, J. (2007). Metodología de las ciencias sociales. Editorial Emecé.

Payarola, M. (2016). Violencia de género: mirada e intervenciones desde la diversidad disciplinar. Multi Group.

Perrone, R. (2012). El síndrome del ángel: consideraciones acerca de la agresividad. Paidós.

Sanmartín, J. (2004). El laberinto de la violencia. Causas, tipos y efectos. Ariel.

Tylor, S., y Bogdan, R. (1987). Introducción a los métodos cualitativos de investigación. Editorial Paidos.

Worchel, S., Cooper, J., Goethals, G. R. y Olson, J. M. (2002). Psicología social. Thomson Editores Paraninfo. 